\title{
Surgery for Primary Tracheal Tumors: Experience on Four Cases
}

\author{
Maruf Sanlia, d, Ersin Arslan ${ }^{\mathrm{a}}$, Ahmet Ferudun Isik ${ }^{\mathrm{a}}$, Bulent Tuncozgur ${ }^{\mathrm{a}}$, \\ Meral Uyarb, Kemal Bakirc ${ }^{\mathrm{c}}$, Levent Elbeyli ${ }^{\mathrm{a}}$
}

\begin{abstract}
Primary tracheal tumors are rare occurrences. The most commonly seen type is the epidermoid carcinoma followed by adenoid cystic carcinoma. Despite being less frequent, schwannomas of primary tracheal origin can also be seen. Of such cases, we had four who had been operated: three for adenoid cystic carcinoma and one for tracheal schwannoma. We analyzed the experiences of our clinic besed on these cases and compared this to those of reported cases. All cases underwent tracheal resection with median sternotomy. One case required additional left hemi-clamshell incision. One case died due to a tracheavascular fistula that developed during the operation. Other cases are at second, third and seventh postoperative years and free of problems. Despite all known risks, the most appropriate type of treatment for primary tracheal tumors is surgery. Radiotherapy is administered as an adjunct treatment to such patients. The rate of fatal complications increases together with the increase in the size of the tracheal segment to be resected.
\end{abstract}

Keywords: Tracheal tumors; Surgical procedures; Operation

\section{Introduction}

The most common type of primary tracheal cancer is epider-

Manuscript accepted for publication September 15, 2010

${ }^{a}$ Gaziantep University, School of Medicine, Department of Thoracic Surgery, Gaziantep

${ }^{\mathrm{b}}$ Gaziantep University, School of Medicine, Department of Pulmonary Diseases, Gaziantep

${ }^{\mathrm{c}}$ Gaziantep University, School of Medicine, Department of Pathology, Gaziantep

${ }^{\mathrm{d}}$ Corresponding author: Gaziantep University School of Medicine Department of Thoracic Surgery, Sahinbey, Gaziantep.

Email: sanli@gantep.edu.tr moid carcinoma (SCC) and it is followed by adenoid cystic carcinoma (ACC). These two histological types constitute $75 \%$ of all primary tracheal malignancies [1]. History of smoking is frequently together with SCC of trachea but no such correlation has been shown for ACC. Clinical course and treatment response of ACC is different than other malignant tumors [2].

Of people having these rare carcinomas, 4 were operated; three had adenoid cystic carcinoma and one had tracheal schwannoma. We analyzed the experience of our clinic based on these four cases and compared this with those of the reported cases.

\section{Case Report}

Four cases were operated for primary tracheal tumors. There were three female patients and one male patient with a mean age of 39.2 years (range 21 - 56 years). None of the patients had a history of smoking. The most common symptoms were dyspnea, cough and hemoptysis. Three patients had a preliminary diagnosis of bronchial asthma and had received related treatment. In the thorax computerized tomography (CT), all patients demonstrated narrowing of the tracheal air column and signs of mass lesions. In the bronchoscopic evaluation, the tumor had cervical localization in one case and thoracal tracheal localization in three cases. Two cases had more than $50 \%$ narrowing and the other two had near total obstructions. All cases underwent tracheal segment resection through median sternotomy and had primary end-to-end anastomoses.

\section{Case 1}

For the last three years, 21-year-old female patient experienced cough, dyspnea and sputum production (of around 1 cup a day) and occasional blood in sputum especially during winter months. Following a flu infection experienced 10 days ago, her complaints increased and the patient admitted to our hospital. In her history, the patient did not report any smoking. In her physical examination, she had widespread inspiratory and expiratory ronchi and clubbing of fingers. In arterial blood gas analysis (ambient air) $\mathrm{pH}$ was $7.46, \mathrm{pO}_{2}$ 
$54.6 \mathrm{mmHg}, \mathrm{pCO}_{2} 33.5 \mathrm{mmHg}$ and $\mathrm{SO}_{2} 90 \%$. In respiratory function tests, FVC was measured as 3.73 (99.1\%), FEV1 $1.13(34.8 \%)$ and FEV1/FVC 35.5. The patient was hospitalized with a preliminary diagnosis of bronchial asthma. In her thorax CT, a nodular opacity of $1 \mathrm{~cm}$ was identified at the superior level of the aortic arcus with protrusion to tracheal lumen. In her bronchoscopy, a lesion was identified 8 $\mathrm{cm}$ distal to the entrance of the trachea; it was protruding to the lumen towards left posterolateral and anterior sides while narrowing it up to $60 \%$. The lesion measured nearly $4 \mathrm{~cm}$ in length; it had a lobulated appearance and its surface was covered with mildly granular mucosa. Pathological examination of the bronchoscopic biopsies obtained from the lesion was reported as adenoid cystic carcinoma. A decision was made to operate the patient. Partial sternotomy and left hemi-clamshell incision starting from 4th intercostal space and extending to anterior axillary line were performed. Pericardium was opened and superior and inferior pulmonary veins were freed. The apex of the heart was elevated and inferior pulmonary ligament was incised. After a tracheal incision performed $1 \mathrm{~cm}$ above the carina close to the distal mar- gin of the lesion, left bronchial system was intubated from the operative field ensuring ventilation. Tracheal segment harboring the lesion was totally excised. Tracheal incision was performed under the guidance of peroperative fiberoptic broncoscopy. Tracheal anastomosis was performed with $2 / 0$ absorbable sutures (PDS, Ethicon) in a continuous fashion obtaining an end-to-end anastomosis. The patient was followed up for a week without any problems, it was provided that she had flexion of the neck and was discharged on postoperative day 8. Pathological specimen contained tumor at its surgical margin. She was referred to radiation oncology for radiotherapy. In control bronchoscopy performed at postoperative second month, anastomosis was solid, tracheal lumen was open and there was minimal fibrosis of the anastomosis line. The patient is at year 7 after the operation and is being followed up without problems.

\section{Case 2}

A 43-year-old female patient was admitted to our clinic after experiencing shorthess of breath for nearly a year and

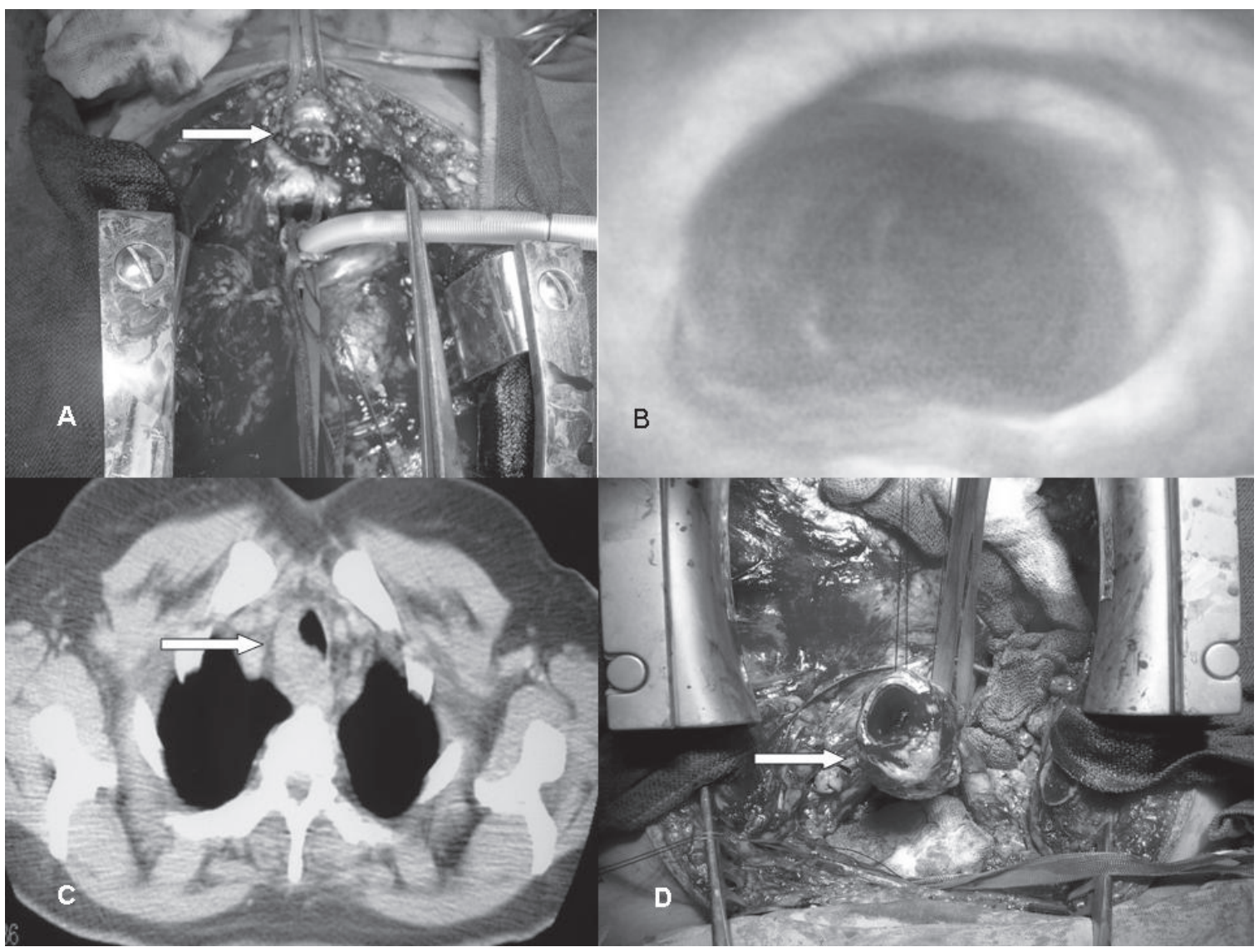

Figure 1. (A) Peroperative appearance of the second case; (B) Control bronchoscopy of the same case was normal; (C) Thorax CT of the third case identified a lesion narrowing the trachea; (D) Resection was performed with median sternotomy. 


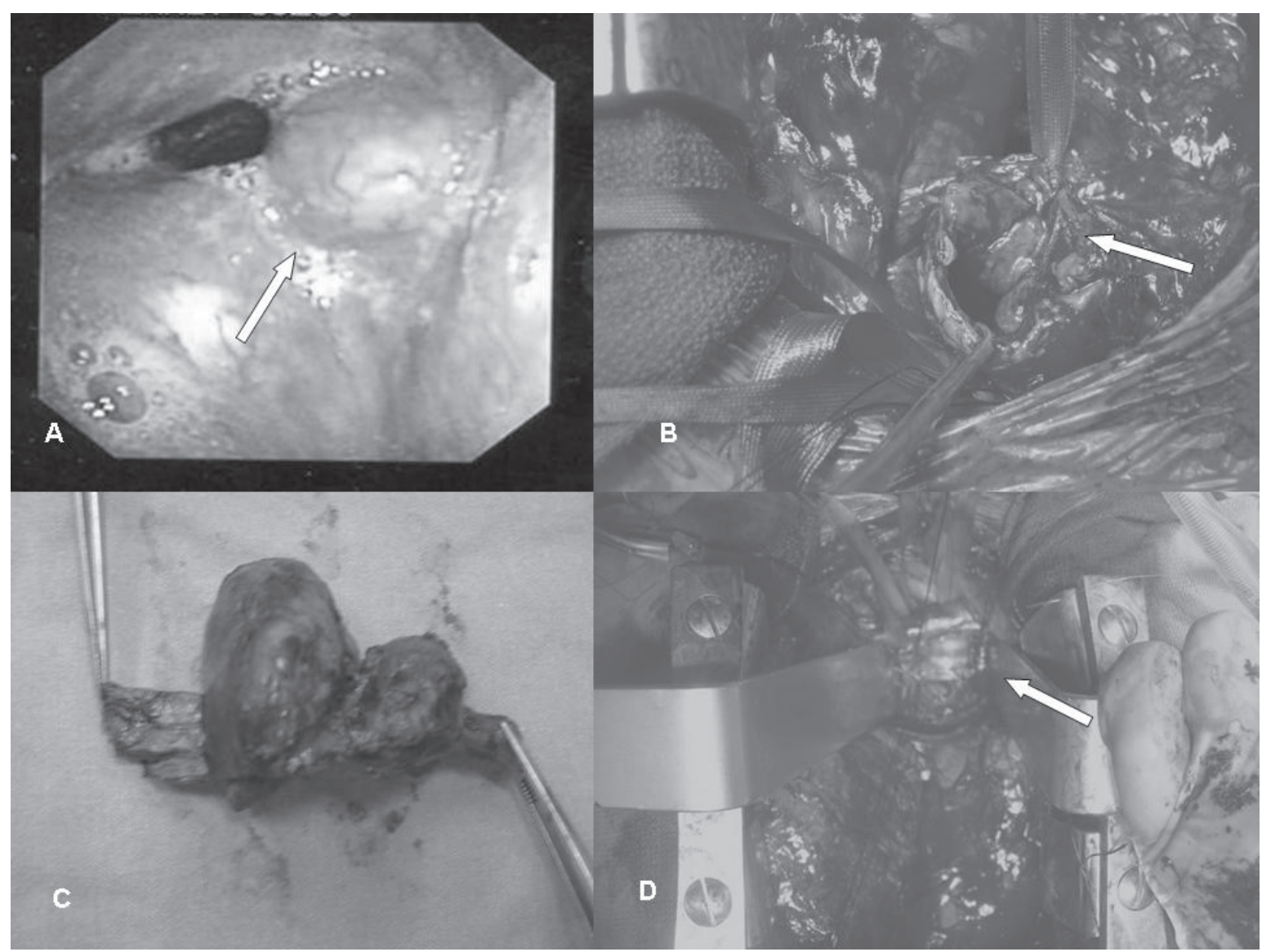

Figure 2. The lesion in the tracheal schwannoma case was described bronchoscopically (A) and intraoperatively (B) after resection (C), tracheal anastomosis was performed (D).

having hemoptysis for about a month. Her history revealed that she was not a smoker and we learned that she has been receiving medical treatment with the diagnosis of bronchial asthma for about a year. In her physical examination, there was stridor. In her bronchoscopy, there was a tumoral mass lesion of about $2 \mathrm{~cm}$ obstructing the lumen about $6 \mathrm{~cm}$ distal to the vocal cords. Pathological examination of the biopsies performed from the lesion was reported as adenoid cystic carcinoma. ESR was $12 \mathrm{~mm} / \mathrm{hr}$. In the analysis of arterial blood gases (with $2 \mathrm{~L} / \mathrm{min}_{2}$ ), $\mathrm{pH}$ was measured as 7.41, $\mathrm{pO}_{2} 175.2 \mathrm{mmHg}, \mathrm{pCO}_{2} 42.9 \mathrm{mmHg}$ and $\mathrm{SO}_{2} 99.5 \%$. A decision was made to operate the patient. Median sternotomy was performed and the trachea was explored. With a $2 \mathrm{~cm}$ circumferential resection performed at the tracheal region below the innominate artery, tracheal segment containing the tumor was excised (Fig. 1A). Tracheal incision was performed with the guidance of fiberoptic bronchoscopy. The tumor was seen to protrude outwards from the membranous wall of the trachea. End-to-end tracheal anastomosis was performed with continuous 3/0 absorbable sutures (Monocrylat, Ethicon). Pediculated mediastinal adipose tissue was wrapped around the anastomosis. Regional lymph node anastomosis was performed. The patient was followed up for a week without problems ensuring neck flexion and was discharged on day 10 . She was referred to radiation oncology to receive radiothreapy. Control bronchoscopies were performed on postoperative day 22 and at month 12 . The anastomosis was found to be solid and the tracheal lumen was open (Fig. 1B). The patient is at month 20 postoperatively and free of problems.

\section{Case 3}

37-year-old female patient having complaints of cough, dyspnea and hemoptysis for about a year was admitted to our clinic. In her history, she had received medical treatment with the diagnosis of bronchial asthma and she did not smoke. In her physical evaluation, she was normal. Thorax CT revealed a mass having lobulated contours with homogeneous contrast enhancement narrowing the trachea at its right lateral border (Fig. 1C). ESR was $7 \mathrm{~mm} / \mathrm{hr}$. Respiratory function tests measured FVC as 2.22 (77\%), FEV1 as 
$1.65(66 \%)$, and FEV1/FVC as 74 . In her bronchoscopy, there was a mass of about $4 \mathrm{~cm}$. It was localized $4 \mathrm{~cm}$ distal to the vocal cords and $4 \mathrm{~cm}$ proximal to the carina. Pathological examination of the bronchoscopic biopsy obtained from the mass was reported as adenoid cystic carcinoma. A decision was made to operate the patient. Following the exploration performed with median sternotomy, pericardium was opened. After hilar freeing, $5 \mathrm{~cm}$ tracheal segment containing the tumor was excised with a peripheral incision (Fig. 1D). Tracheal incision was performed with the help of peroperative fiberoptic bronchoscopy. Tracheal end-to-end anastomosis was performed with continuous 3/0 absorbable sutures (PDS, Ethicon) and regional lymph node dissection was performed. The patient was being followed up by ensuring neck flexion; however, she developed subcutaneous emphysema on postoperative day 5. Bilateral pleural effusion that was identified on control thorax CT was drained with a thin catheter. In control fiberoptic bronchoscopy, the patient had partial separation of the anastomosis and she died of abrupt tracheal hemorrhage (tracheovascular fistula) on postoperative day 11 .

\section{Case 4}

56-year-old male patient experiencing dry cough and fatigue for the last two months was admitted to our clinic. His history was not unusual and his phycial examination was normal. ESR was $3 \mathrm{~mm} / \mathrm{hr}$. On thorax CT, there was a solid noduler lesion of $3 \times 2 \mathrm{~cm}$ with regular borders located $3 \mathrm{~cm}$ proximal to the level of bifurcation while neighboring the trachea posteriorly. In his bronchoscopy, proximal trachea was normal. At the distal level, there was a nodular mucosal lesion covered with mucosa with significant vascularity and a shiny surface $3 \mathrm{~cm}$ proximal to the carina. The lesion caused narrowing of the lumen from lateral and posterior thereby creating an hour glass appearance (Fig. 2A). Pathological examination of the bronchoscopic biopsies obtained from the tracheal lesion was reported as mesenchymal tumor. A decision was made to operate the patient. Median sternotomy was used for entry. The part of the trachea harboring the tumoral mass was excised $3 \mathrm{~cm}$ circumferentially (Fig. 2B, 2C). Peroperative fiberoptic bronchoscopy performed during the excision provided guidance. End-to-end tracheal anastomosis was performed with continuous 3/0 absorbable sutures on the posterior part (PDS, Ethicon) and with single 3/0 absorbable sutures (Vicryl, Ethicon) that were tied outside for lateral and anterior parts (Fig. 2D). Regional lymph node dissection was performed. Thymic tissue was covered on the anastomosis. The patient was followed up for a week without problems ensuring neck flexion and was discharged on postoperative day 8 . After a long follow-up, the pathological material that was excised was reported as schwannoma. Control bronchoscopy was performed on postoperative day 15. Anastomosis was found to be solid and tracheal lu- men was open, yet there was mucosal wall irregularity on the anastomosis line. The patient is at the 26 th postoperative month and free of any complaints.

\section{Discussion}

Of our 4 patients, three had ACC and one had schwannoma. Treatment was achieved with circumferential tracheal resection through median sternotomy and end-to-end anastomosis. There was no gender preference in other report for ACC [2] but all of our cases were women. As expected, patients with ACC did not smoke.

Most common symptoms were dyspnea, hemoptysis and cough. Other symptoms were dysphagia, weight loss, stridor, sore throat and wheezing. In order for the dyspnea that is significant during exertion to appear, there needs to be significant airway narrowing ( $75 \%$ obstruction). Due to their slow developmental pattern and silent nature, tracheal tumors can remain unidentified for months and even for years. Furthermore, these patients can frequently be treated for asthma or other erroneous diagnoses due to chronic cough, hoarseness and difficulties in expelling the secretions [3]. Three of our cases had histories of medical treatment due to diagnoses of asthma. Specifically, in patients having unsuccessful treatment histories with symptoms like dyspnea, cough and hemoptysis after having a preliminary diagnosis of asthma, differential diagnosis should include primary tracheal cancer.

In chest radiography, tracheal tumors are located in relatively blind fields. That is why the diagnosis is delayed until the tumor is identified via CT or bronchoscopy. Chest radiogram reveals abnormalities in only $1 / 3$ of the patients and laryngoscopy in only $25 \%$, therefore airway CT or bronchoscopy should be performed for patients experiencing diagnostic problems. Bronchoscopy does not only serve diagnostic purposes, it can be used for the palliation of the airways after diminishing the tumor size or placement of stents in selected unresectable patients [2]. Thorax CT identified narowing of the tracheal air column in all patients. Bronchoscopic examination was performed in all cases and proximal half of the trachea was identified as cervical while distal half was identified as thoracic. As shown by bronchoscopic examination, one case had cervical and three cases had thoracal tracheal localization. Three cases had more than 50\% narrowing of the lumen and one patient had near total obstruction.

Treatment of choice for primary tracheal cancers includes surgical resection; however this modality is not free of risks or sequelae [2]. Radiotherapy is appropriate as an adjunct but its use by itself is not successful. Brachytherapy, photodynamic treatment and criotherapy are other alternatives used for palliative purposes [4].

Although complete resection is the preferred treatment for adenoid cystic carcinomas, it is not usually possible to perform this due to delays in the diagnosis. It is difficult to 
ensure the negativity of the surgical margins due to spreading of the tumors alongside submucosal and perineural structures [5]. In resections of tracheal segment that are longer than $6 \mathrm{~cm}$, complete resection might not be possible due to anastomotic insufficiency [6]. However, the prognoses of ACC patients who undergo surgery are more favorable than those with squamous carcinomas. For most cases, adjunct radiotherapy together with surgery is the preferred treatment [4]. Instead of making a resection that would leave the anastomosis under tension, one could accept microscopic tumor positivity at the surgical margin as experienced in case 1 and could recommend radiotherapy.

Primary tracheal schwannoma is less common than ACC. For such cases, complete resection should be the preferred surgical option. Recurrences are rare after complete resections and prognoses of such cases are favorable [7]. Case 4 underwent complete resection for tracheal schwannoma and is being followed up without recurrences in postoperative year 3 .

For cases requiring large tracheal segment resections, different tracheal freeing manuevers are employed. During median sternotomy, such approaches necessitate upward rotation of the cardiac apex. Such manipulations might lead to hemodynamic disturbances and cardiac problems. In patients in need of large tracheal resections, limited operative field can be enlarged by complementing partial median sternotomy with hemi-clamshell incision to serve the need for manipulations geared at freeing as performed in case 1. In tracheal surgery, cervical approach is preferred for lesions with cervical localization, and right posterolateral thoracotomy incision to be performed from fourth intercostal space is recommended for lesions with thoracic localizations. If partial median sternotomy was to complement the cervical incision, this would allow for interventions throughout the full length of the trachea. Median sternotomy was used in all of our cases. Under the guidance of a flexible peroperative bronchoscope, tracheal segment resection and anastomoses were performed.

Meticulous surgical planning and employment of freeing procedures allow for secure resections of nearly $50 \%$ of the tracheal length in selected patients [4]. During recent years, the possibility of using tracheal transplants through studies in related tissue engineering [8] is promising for the purpose of long tracheal segment resections. However, as anastomotic separation has a fatal course, if closure would not be possible without creating a tension, surgical resection should not be attempted at all. In anastomoses performed under tension or in cases with disrupted tracheal vascular support during dissection, separation of the tracheal anastomosis can develop. By giving rise to infections, such complications can have fatal courses as experienced by a tracheavascular fistula resulting in death in our third case.

In conclusion, for primary tracheal tumors, surgical resection is the treatment of choice. Together with the increases in the size of the segment to be resected, the rate of fatal complications will increase. For cases of adenoid cystic carcinoma, resections allowing for secure anastomoses and postoperative radiotherapy would be the proper approach.

\section{References}

1. Grillo HC. Development of tracheal surgery: a historical review. Part 2: Treatment of tracheal diseases. Ann Thorac Surg 2003;75(3):1039-1047.

2. Webb BD, Walsh GL, Roberts DB, Sturgis EM. Primary tracheal malignant neoplasms: the University of Texas MD Anderson Cancer Center experience. J Am Coll Surg 2006;202(2):237-246.

3. Azar T, Abdul-Karim FW, Tucker HM. Adenoid cystic carcinoma of the trachea. Laryngoscope 1998;108(9):1297-1300.

4. Meyers BF, Mathisen DJ. Management of Tracheal Neoplasms. Oncologist 1997;2(4):245-253.

5. Clough A, Clarke P. Adenoid cystic carcinoma of the trachea: a long-term problem. ANZ J Surg 2006;76(8):751753.

6. Gaissert HA, Grillo HC, Shadmehr MB, Wright CD, Gokhale M, Wain JC, Mathisen DJ. Long-term survival after resection of primary adenoid cystic and squamous cell carcinoma of the trachea and carina. Ann Thorac Surg 2004;78(6):1889-1896; discussion 1896-1887.

7. Righini CA, Lequeux T, Laverierre MH, Reyt E. Primary tracheal schwannoma: one case report and a literature review. Eur Arch Otorhinolaryngol 2005;262(2):157160.

8. Macchiarini P, Jungebluth P, Go T, Asnaghi MA, Rees LE, Cogan TA, Dodson A, et al. Clinical transplantation of a tissue-engineered airway. Lancet 2008;372(9655):20232030. 